\title{
Attraction of Brachyspira pilosicoli to mucin
}

\author{
Correspondence \\ David J. Hampson \\ d.hampson@murdoch.edu.au
}

Received 22 April 2009

Revised 14 October 2009

Accepted 14 October 2009

\author{
Ram Naresh and David J. Hampson
}

Animal Research Institute, School of Veterinary and Biomedical Science, Murdoch University, Murdoch, Western Australia 6150, Australia

\begin{abstract}
The anaerobic intestinal spirochaete Brachyspira pilosicoli colonizes the large intestine of various species, including humans. In the colon this spirochaete can penetrate the overlying mucus layer, attach by one cell end to the underlying enterocytes, and initiate localized colitis and diarrhoea. The aim of this study was to investigate whether, as part of the colonization process, B. pilosicoli is attracted to mucin. Fifteen B. pilosicoli strains isolated from humans, pigs, chickens and dogs, and a control strain of Brachyspira hyodysenteriae, were analysed for their ability to enter solutions of hog gastric mucin in an in vitro capillary tube assay. No significant attraction was detected with $1 \%$ mucin, but some strains started to enter a $2 \%$ solution, and attraction then increased with increasing concentrations to peak at around 6-8\% mucin. A similar increase was seen with $B$. hyodysenteriae, although this activity peaked at $6 \%$ mucin and then declined, suggesting that the two species have different affinities for mucin. These mucin concentrations were much higher than those used in previous experimental studies with Brachyspira species. The viscosities of the $6-8 \%$ mucin solutions were around 7-12 $\mathrm{mPa} \mathrm{s}$, which were similar to the measured viscosities of the mucus layer overlying the epithelium in the caecum and colon of experimental pigs. The strains varied in their motility, as assessed by their ability to enter tubes containing chemotaxis buffer, but there was no significant relationship between this motility and the extent of their ability to enter the mucin solutions. Different strains also had different propensities to enter the mucin solutions, but there were no consistent differences according to the host species of origin. B. pilosicoli strain 95/1000 was attracted towards a solution of Dserine, suggesting that chemotaxis was involved in the attraction to mucin; however, 95/1000 was also attracted to viscous solutions of polyvinylpyrrolidone (PVP), in a manner mirroring the response to mucin, and hence suggesting the involvement of viscotaxis in the attraction to mucin. B. hyodysenteriae B204 showed a similar viscotaxis to PVP. Further studies are required to determine whether the in vitro interaction of a given strain with mucin is a useful indicator of its in vivo colonization ability, and hence could be used as a potential marker for virulence.
\end{abstract}

\section{INTRODUCTION}

The anaerobic intestinal spirochaete Brachyspira pilosicoli colonizes the large intestine of various species of animals and birds, as well as human beings (Hampson et al., 2006). A feature of the colonization is the intimate end-on attachment of spirochaete cells to the luminal surface of colonic and rectal epithelial cells, in a condition called 'intestinal spirochaetosis' (IS). Infections with B. pilosicoli are common amongst intensively farmed pigs and chickens, in which the spirochaete is considered to be an important enteric pathogen (Stephens \& Hampson, 2001; Hampson \& Duhamel, 2006), but it is also common in people living in crowded and unhygienic conditions in developing countries (Trott et al., 1997a; Margawani et al., 2004; Munshi et al., 2004), and in homosexual males and HIV patients in developed countries (Law et al., 1994;

Abbreviations: PVP, polyvinylpyrrolidone; $R_{\text {att, }}$ attraction ratio.
Trivett-Moore et al., 1998). Based on the apparent panmictic population structure of the species, and the distribution of $B$. pilosicoli strains isolated from different host species on dendrograms produced from multilocus enzyme electrophoresis data, it has been suggested that there is unlikely to be any major barrier to zoonotic transmission of B. pilosicoli strains (Trott et al., 1998; Hampson et al., 2006).

In order to be able to colonize and attach to colonic enterocytes, $B$. pilosicoli cells need to penetrate and move through the overlying mucus layer. In mice, and presumably other species, the mucus gel overlying colonic enterocytes forms two layers, with the innermost layer being more densely packed than the outer layer, and devoid of the commensal bacteria that are normally found in the outer layer (Johansson et al., 2008). Spirochaete cells have a corkscrew-like motility, allowing them to advance through highly viscous substances such as mucus (Li et al., 2000). It 
is not known whether B. pilosicoli can disrupt the mucus layer, for example by enzymic degradation (Lidell et al., 2006), but in any case the spirochaete presumably requires a high degree of motility and swimming efficacy to be able to penetrate the mucus layers. In addition to motility, chemotaxis is known to be an important virulence factor in many pathogenic spirochaete species, allowing them to target the relevant colonization sites (Lux et al., 2000). Consistent with this, virulent strains of the related intestinal spirochaete Brachyspira hyodysenteriae (the aetiological agent of swine dysentery) have been reported to be chemotactic to mucin, the major component of the mucus gel, as well as to components of mucin such as Lserine (Kennedy \& Yancey, 1996). On the other hand, avirulent strains of $B$. hyodysenteriae were found to have reduced chemotaxis to mucin compared to virulent strains such as B204 (Milner \& Sellwood, 1994). In contrast, and unexpectedly, in the latter study analysis of five porcine $B$. pilosicoli strains showed that these were not attracted to mucin under the same test conditions as B. hyodysenteriae (Milner \& Sellwood, 1994), despite B. pilosicoli clearly needing to be able to penetrate the mucous layers to access attachment sites. More recently, Witters \& Duhamel (1999) investigated three $B$. pilosicoli strains, and found that one from a human being and one from a dog were slightly attracted to $1 \%$ porcine gastric mucin, whilst a third porcine strain that had also been examined by Milner \& Sellwood (1994) was not attracted to mucin, but it was mildly attracted to $10 \mathrm{mM}$ DL-serine.

The aim of the current study was to investigate the extent to which B. pilosicoli is attracted to mucin, and to determine whether this property differs amongst strains of the species. Comparisons were also made with the behaviour of B. hyodysenteriae strain B204, and the underlying basis of the strong attraction to mucin that was found was investigated.

\section{METHODS}

Animal ethics. Permission to use the pigs in this study was obtained from the Murdoch University Animal Ethics Committee.

Spirochaete strains and culture conditions. Brachyspira strains were obtained as frozen stock from the culture collection held at the Australian Reference Centre for Intestine Spirochaetes at Murdoch University. They included 15 strains of B. pilosicoli (five isolated from humans, four from pigs, and three each from chickens and dogs), and Brachyspira hyodysenteriae strain B204. Of these strains, B. pilosicoli porcine strain $\mathrm{P} 43 / 6 / 78^{\mathrm{T}}$ had previously been examined for chemotaxis to porcine gastric mucin by both Milner \& Sellwood (1994) and Witters \& Duhamel (1999), and canine strain 16242-94 had previously been examined by Witters \& Duhamel (1999). B. hyodysenteriae strain B204 had been examined for chemotaxis to mucin by Milner \& Sellwood (1994) and by Kennedy \& Yancey (1996).

The spirochaete cells were thawed and grown in Kunkle's pre-reduced anaerobic broth, containing $2 \%(\mathrm{v} / \mathrm{v})$ fetal bovine serum and $1 \%$ $(\mathrm{v} / \mathrm{v})$ ethanolic cholesterol solution (Kunkle et al., 1986). Broth cultures were incubated at $39{ }^{\circ} \mathrm{C}$ on a rocking platform for $3-5$ days, and spirochaete growth and motility was monitored daily by examining aliquots under a phase-contrast microscope. Only actively motile mid-exponential-phase spirochaete cells were used in the assays. Cells were counted using a Neubauer counting chamber under the $40 \times$ objective of a phase-contrast microscope, and final dilutions to $10^{8}$ cells $\mathrm{ml}^{-1}$ were made in freshly prepared pre-warmed anaerobic broth.

Capillary tube assays. The system used was based on the method of Milner \& Sellwood (1994), with modifications. Porcine gastric mucin type II (Sigma Aldrich) was dissolved in chemotaxis buffer [0.01 M potassium phosphate buffer ( $\mathrm{pH} 7.0$ ), $0.2 \mathrm{mM}$ L-cysteine hydrochloride] (Greenberg \& Canale-Parola, 1977) to final concentrations of $10,8,6,4,3,2,1,0.5$ and $0.2 \%$ (w/v). D-Serine (Sigma) was similarly dissolved in chemotaxis buffer to make 100,10, 1 and $0.1 \mathrm{mM}$ solutions, and polyvinylpyrrolidone (PVP) (Sigma) was dissolved in chemotaxis buffer to make solutions of 4.0, 3.0, 2.5, 2.0, $1.5,1.25,0.75$ and $0.25 \%(\mathrm{w} / \mathrm{v})$.

Only B. pilosicoli strain $95 / 1000$ and B. hyodysenteriae strain B204 were tested in 8 and $10 \%$ mucin, whilst all strains were tested in the other seven dilutions of mucin. Strain 95/1000 was also tested in PBS (per litre: $10 \mathrm{~g} \mathrm{NaCl}, 0.25 \mathrm{~g} \mathrm{KCl}, 0.1438 \mathrm{~g} \mathrm{Na}_{2} \mathrm{HPO}_{4}, 0.25 \mathrm{~g} \mathrm{KH}_{2} \mathrm{PO}_{4}$; $\mathrm{pH} 7.2$ ), in chemotaxis buffer and in the D-serine solutions, and it and B204 were tested in the various dilutions of PVP, with $6 \%$ mucin used as a positive control. A suspension of strain $95 / 1000$ that had been inactivated overnight in $4 \%$ formalin was also tested with chemotaxis buffer and $4 \%$ mucin. For each spirochaete strain to be tested, six $75 \mathrm{~mm}$ haematocrit capillary tubes (75 $\mu \mathrm{l}$ volume; Hirschmann Laborgerate) were filled with the appropriate concentration of mucin or the various other solutions, and six paired tubes were filled with chemotaxis buffer. The top ends of the tubes were sealed with Plasticine, and then the tubes were hung vertically with their lower ends submerged in fresh bacterial broth culture $\left(10^{8}\right.$ cells $\mathrm{ml}^{-1}$ ) in 48-well round-bottomed tissue culture plates. These were incubated at $39{ }^{\circ} \mathrm{C}$ in a $\mathrm{CO}_{2}$ incubator. An appropriate incubation time was determined by testing B. pilosicoli strain $95 / 1000$ in $4 \%$ mucin in chemotaxis buffer with incubation times of 15, 30, 60, 90 and $120 \mathrm{~min}$. Based on the results, a $90 \mathrm{~min}$ incubation time was selected for testing all the strains. After incubation, each tube was carefully removed, the outside wiped dry with a sterile tissue, and then placed upright into a $200 \mu$ l Eppendorf tube. The top of the capillary tube was gently broken and the contents were collected in the tube. The collected solution was serially diluted in PBS and the spirochaetes were counted under a phase-contrast microscope, as described above.

Viscosity measurements. Six healthy pigs of $\sim 30 \mathrm{~kg}$ body weight, that had been fed on a commercial antimicrobial-free Australian grower diet based on wheat and lupins, were killed using a captive bolt pistol and exsanguination, and their large intestines were removed and opened. The intestinal contents were expelled manually, the mucosa rinsed with PBS, patted dry with paper tissue, and approximately $1 \mathrm{~g}$ quantities of the mucus layer immediately overlying the epithelium in both the mid-caecum and proximal colon were carefully collected by gently scaping with sterile scalpel blades. The viscosities of these samples were measured in a Brookfield LVDV-II + cone plate $(\mathrm{CP} 40)$ rotational viscometer (Brookfield Engineering Laboratories), as previously described (Hopwood et al., 2002). Briefly, the material was diluted $1: 1(\mathrm{v} / \mathrm{v})$ with distilled water, mixed and centrifuged at $12000 \mathrm{~g}$ for $8 \mathrm{~min}$. The viscosities of $0.5 \mathrm{ml}$ fractions held at $39{ }^{\circ} \mathrm{C}$ in a water bath were measured, applying a shear rate of $60 \mathrm{~s}^{-1}$. The viscosities of the dilutions of porcine gastric mucin and PVP used in the capillary tube assays were similarly tested.

Statistical analysis. For each replicate of each mucin concentration or other solution tested, attraction was expressed as the ratio $\left(R_{\mathrm{att}}\right)$ of 
the number of spirochaetes in the tube containing the test solution compared to the number in the paired tube containing chemotaxis buffer. The mean and standard deviation of the six replicates for each concentration were calculated. One-way analysis of variance (ANOVA) was used to compare the $R_{\text {att }}$ for each of the 15 strains across the different mucin concentrations, to compare the $R_{\text {att }}$ for each of the 15 strains at each of the seven mucin concentrations, and to compare the $R_{\mathrm{att}}$ at each of the mucin concentrations for the sets of isolates from humans, pigs, chickens and dogs. $R_{\text {att }}$ values of $>2$ were considered significant (Moulton \& Montie, 1979). $R_{\mathrm{att}}$ values of zero were obtained when no spirochaetes were found in the mucin solution, but spirochaetes were present in the buffer tubes. Comparison of the number of spirochaetes in the capillary tubes containing only chemotaxis buffer for the 15 strains were similarly analysed by ANOVA. Regression analyses were made across all the strains for the numbers of spirochaetes in the tubes containing chemotaxis buffer and the $R_{\text {att }}$ values in $4 \%$ and in $6 \%$ mucin.

\section{RESULTS}

\section{Optimizing test conditions}

The responses of B. pilosicoli strain $95 / 1000$ and $B$. hyodysenteriae strain B204 to various mucin concentrations from 0.2 to $10 \%$ are presented in Fig. 1 . In both species the $R_{\text {att }}$ value increased with increasing mucin concentrations, to peak at around $6-8 \%$ mucin for $95 / 1000$, and $6 \%$ for B204, after which it showed a steep decline for the $B$. hyodysenteriae strain. Based on these results, all the $B$. pilosicoli strains were tested in mucin concentrations from $0.2 \%$ to $6 \%$. The results of incubating strain $95 / 1000$ in $4 \%$ mucin for different time periods are shown in Fig. 2. Based on these findings, an incubation time of 90 min was selected for all further incubations.

Chemotaxis buffer supported the activity of $B$. pilosicoli better than did PBS, as significantly $(P<0.001)$ more cells of strain 95/1000 were found in the tubes containing chemotaxis buffer than in those containing PBS (2.97 and $1.56 \times 10^{6}$ cells ml $^{-1}$, respectively). Cells of B. pilosicoli 95/ 1000 that had been inactivated in formalin did not enter the tubes containing chemotaxis buffer or mucin.

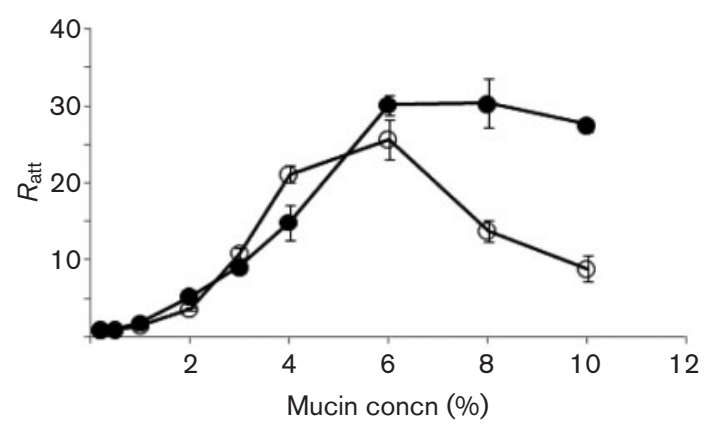

Fig. 1. $R_{\text {att }}$ values (means $\pm \mathrm{SD}$ ) for $B$. pilosicoli strain $95 / 1000$ $(\bullet)$ and $B$. hyodysenteriae strain B204 $(\bigcirc)$ incubated for $90 \mathrm{~min}$ in different concentrations of porcine gastric mucin in chemotaxis buffer.

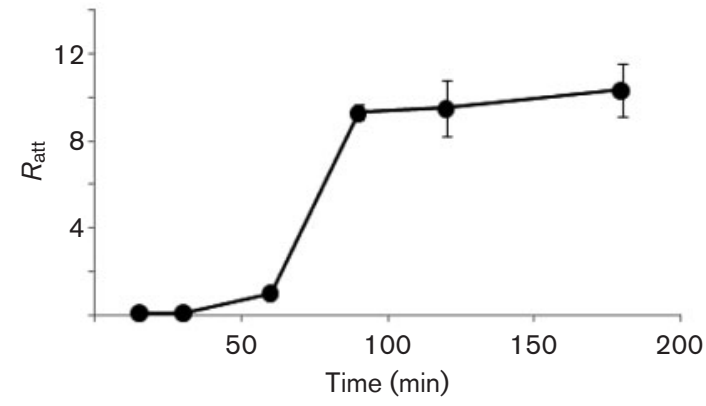

Fig. 2. $R_{\text {att }}$ values (means $\pm \mathrm{SD}$ ) for $B$. pilosicoli strain $95 / 1000$ incubated for $15,30,60,90,120$ and $180 \mathrm{~min}$ in $4 \%$ mucin.

When analysing the B. pilosicoli numbers in the tubes containing only chemotaxis buffer after 90 min incubation, significant differences in counts were found for the different spirochaete strains $(P<0.001$; Table 1$)$. The corresponding mean number of cells for B. hyodysenteriae B204 in chemotaxis buffer was $(2.09 \pm 0.28) \times 10^{6}$. When the numbers of $B$. pilosicoli cells in chemotaxis buffer were compared by linear regression with their $R_{\text {att }}$ values for the same strains in $4 \%$ and $6 \%$ mucin, no significant associations were found $(P=0.738$ and $P=0.401$, respectively).

\section{$\boldsymbol{R}_{\text {att }}$ values of different spirochaete strains with mucin}

The means and standard deviations of the $R_{\mathrm{att}}$ values for the 15 B. pilosicoli strains at seven mucin concentrations are presented in Table 1. For each strain the different $R_{\mathrm{att}}$ values across the mucin concentrations all showed significant differences $(P<0.0001)$. The results for individual strains varied considerably. Strain Cof-10, isolated from a pig, was not attracted to any concentration of mucin, and indeed it was unable to enter solutions of $1 \%$ mucin or more. Strain 16242.94, isolated from a dog, showed the greatest attraction. All comparisons of the $R_{\mathrm{att}}$ for the 15 strains at all the mucin concentrations showed statistically significant differences $(P<0.0001)$. Comparisons of sets of strains based on the four different species of origin revealed no significant differences at any mucin concentration. For mucin concentrations up to and including $1 \%$, no strains had a $R_{\text {att }}$ value $>2$. At mucin concentrations of $2 \%$, three human, one porcine and two canine strains of B. pilosicoli had $R_{\text {att }}$ values $>2$. At $3 \%$ mucin all strains except Cof- 10 and L72 had $R_{\text {att }}$ values $>2$, and at $4 \%$ and $6 \%$ mucin, only Cof- 10 did not show evidence of attraction to mucin. For nearly all strains the attraction increased with increasing mucin concentrations, up to $6 \%$. The $R_{\text {att }}$ values for some B. pilosicoli strains with $6 \%$ mucin were extremely high, and exceeded those for $B$. hyodysenteriae B204 (Fig. 1).

\section{$\boldsymbol{R}_{\text {att }}$ values of $\mathbf{9 5 / 1 0 0 0}$ with D-serine}

B. pilosicoli strain $95 / 1000$ showed a significant $R_{\text {att }}$ $(2.58 \pm 0.55)$ with the $100 \mathrm{mM}$ solution of D-serine only. 
Table 1. $R_{\text {att }}$ values at seven concentrations of porcine gastric mucin, and the number of spirochaete cells in chemotaxis buffer tubes, for 15 strains of $B$. pilosicoli after 90 min incubation

Means \pm SD are shown. Values indicating significant attraction to mucin $\left(R_{\mathrm{att}}>2\right)$ are in bold.

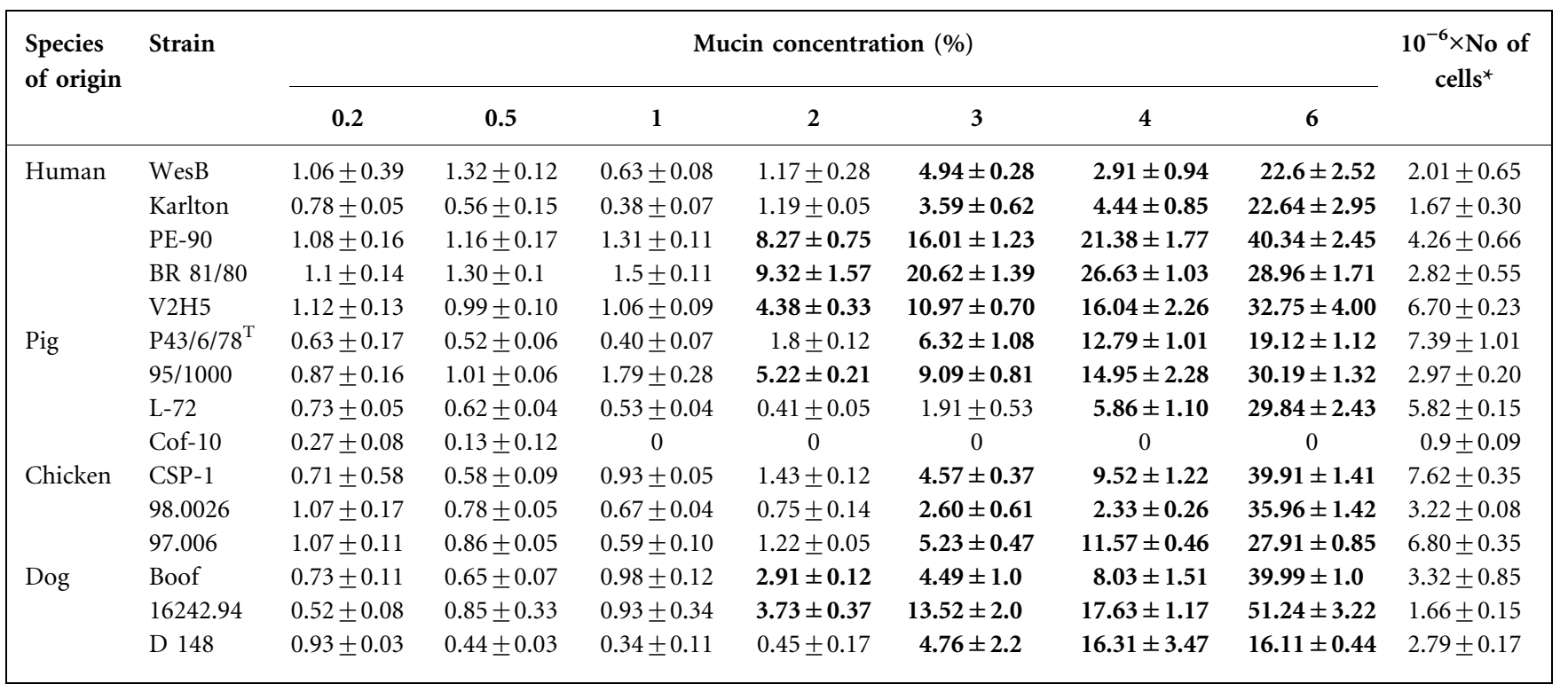

*Spirochaete cell numbers in tubes containing only chemotaxis buffer.

\section{$R_{\text {att }}$ values of $95 / 1000$ and B204 in relation to viscosity}

The viscosities of the mucin and PVP dilutions are listed in Table 2. The $R_{\text {att }}$ results in these solutions in relation to their viscosity for B. pilosicoli $95 / 1000$ and B. hyodysenteriae B204 are shown in Fig. 3. For 95/1000, the $R_{\text {att }}$ increased with increasing PVP concentrations to plateau from a viscosity of around $6 \mathrm{mPa} \mathrm{s}$ (Fig. 3a). These $R_{\text {att }}$ values followed the same trend as seen with mucin at the same viscosities, but at a given viscosity were only about $20 \%$ of the values obtained with mucin (Fig. 3b). For B204, the $R_{\text {att }}$ continued to increase with increasing viscosities of the PVP solutions, although the $R_{\text {att }}$ had declined in the mucin solutions at the (similar) high viscosities. As with $95 / 1000$,

Table 2. Viscosities of the dilutions of mucin and PVP used in the attraction assays

\begin{tabular}{|lc|cc|}
\hline Mucin (\%) & Viscosity (mPa s) & PVP (\%) & Viscosity (mPa s) \\
\hline 0.2 & 1.2 & 0.25 & 1.5 \\
0.5 & 1.3 & 0.75 & 2.1 \\
1.0 & 1.4 & 1.25 & 3.5 \\
2.0 & 1.6 & 1.5 & 4.3 \\
3.0 & 1.9 & 2.0 & 5.8 \\
4.0 & 2.9 & 2.5 & 7.8 \\
6.0 & 7.2 & 3.0 & 10.8 \\
8.0 & 12.4 & 4.0 & 16.9 \\
10.0 & 13.6 & & \\
& & & \\
\hline
\end{tabular}

the $R_{\text {att }}$ values obtained with B204 generally were much higher in the mucin solutions than in the PVP solutions; however, at the higher viscosities the $R_{\mathrm{att}}$ in mucin declined to values similar to those in the PVP solutions.
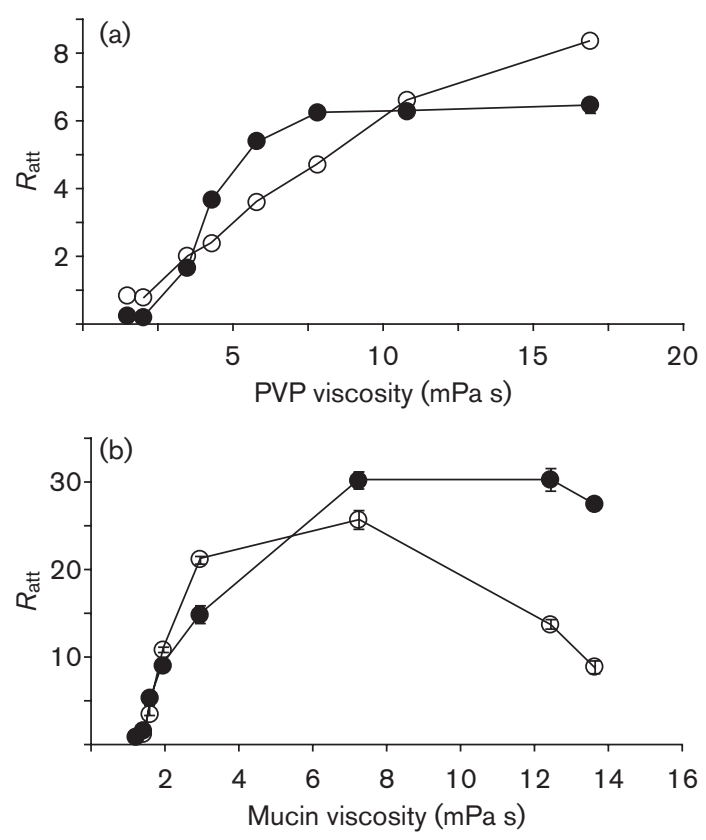

Fig. 3. $R_{\text {att }}$ values obtained for $B$. pilosicoli $95 / 1000(0)$ and $B$. hyodysenteriae B204 ( $\bigcirc)$ in dilutions of PVP (a) and mucin (b), plotted against measured viscosities. 


\section{Viscosity of caecal and colonic mucus}

The mucus layer overlying the epithelium in the porcine caecum and colon had viscosities of $7.53 \pm 1.89$ and $7.16 \pm 2.21 \mathrm{mPa} s$, respectively. These values were similar to those of the $6 \%$ mucin concentration (Table 2).

\section{DISCUSSION}

In optimizing the capillary tube assay, the chemotaxis buffer supported the motility of the spirochaetes better than did PBS, and this is in agreement with previous findings (Milner \& Sellwood, 1994). On the other hand, an incubation period of $90 \mathrm{~min}$ was preferable to the incubation period of $60 \mathrm{~min}$ that has previously been used in similar experiments (Milner \& Sellwood, 1994; Witters \& Duhamel, 1999), and hence this was selected for use throughout the study. The use of a shorter incubation time by previous investigators might have underestimated the extent of the attraction to mucus that the previous workers recorded for a given strain.

A striking and important finding early in this study was the observation that the attraction of B. pilosicoli strain 95/1000 and B. hyodysenteriae strain B204 increased with increasing mucin concentrations. Previous studies on attraction to mucin by B. pilosicoli and B. hyodysenteriae have used $1 \%$ mucin, and it is clear that this concentration is not optimal for assessing how the spirochaetes are attracted to mucin. Indeed, in the current study no strains showed significant attraction to $1 \%$ mucin. These differences in the assay conditions help to explain the discrepancies in the results in the current study with those of the previous studies.

A factor potentially influencing the viability of the spirochaetes and their attraction to mucin is the medium and conditions under which they are grown. For example, Witters \& Duhamel (1999) found that two B. pilosicoli strains only showed attraction to mucin if they were grown in a defined medium containing either pig faecal extract or $0.1 \%$ mucin. The influence of the culture medium was not investigated in the current study, as clear responses were obtained under the standard culture conditions that were used.

Another important finding in the study was that attraction to mucin varied considerably between $B$. pilosicoli strains, with one of the 15 strains (Cof-10) not showing any attraction. Furthermore, the spirochaete strains varied in their capacity to enter the chemotaxis buffer, and this was taken to indicate that they also varied in their intrinsic motility under a given set of environmental conditions. Although Cof-10 was the least motile strain, and it was not attracted to mucin, over all the strains greater or lesser 'basal' motility did not necessarily equate with greater or lesser attraction to mucin. In vivo, the capacity to efficiently colonize and cause disease presumably requires both motility and strong attraction to mucin. Cof-10 was isolated from an Australian pig as part of a prevalence survey, but its ability to cause disease has not been investigated experimentally. On the other hand, B. pilosicoli strains WesB, P43/6/78 ${ }^{\mathrm{T}}, 95 / 1000$ and CSP-1 as well as other strains have been used successfully to experimentally infect pigs and/or chickens (Taylor et al., 1980; Trott et al., 1995, 1996; Stephens \& Hampson, 2002). These strains were all found to be motile and to be attracted to mucin in the current study, although they differed in the extent of this activity. Ideally, strains with known attraction and motility properties should be tested simultaneously in an animal model to determine whether the in vitro measurements equate with in vivo colonization capacity, and pathogenic potential. It was interesting that the human strains PE-90 and BR 81/80, isolated from the bloodstream of French patients (Trott et al., 1997b), were strongly attracted to mucin. This attribute may have facilitated their colonization of the large intestine in these patients, and increased opportunities for their translocation through the colonic epithelium.

In this study there was no significant correlation between the species from which a given strain of B. pilosicoli was isolated and the extent of its attraction to mucin, or motility. This is consistent with previous observations that strains of $B$. pilosicoli from humans do not form a genetically distinct group from strains recovered from animals (Hampson et al., 2006). Strains from all sources varied in their properties, and this supports the likelihood that cross-species transmission of B. pilosicoli strains can occur.

Mucin is a serine-threonine-proline-rich protein with covalently bound polysaccharides, and such amino acids and polysaccharides can bind to chemotaxis receptors on the surface of various bacteria, including spirochaetes (Lux et al., 2000). Previously B. pilosicoli strain $\mathrm{P} 43 / 6 / 78^{\mathrm{T}}$ has been shown to be chemotactic to DL-serine (Witters \& Duhamel, 1999), a component of mucin, and a similar response to $\mathrm{D}$-serine was obtained in the current study with strain $95 / 1000$. Hence it appears likely that the attraction to mucin by $B$. pilosicoli includes an element of chemotaxis. Other potential attractants that would be present in mucin and the mucous gel include a range of simple sugars, and further work is required to identify these. Previously $B$. hyodysenteriae has been shown to be chemotactic towards a variety of mucin components (Kennedy \& Yancey, 1996), and $B$. hyodysenteriae is known to have a large number of genes encoding methyl-accepting chemotaxis proteins (46) and chemosensory transductor molecules (17) (Bellgard et al., 2009). Less is known about B. pilosicoli, although one putative glucose-galactose chemoreceptor has been described (Zhang et al., 2000). It is not clear how chemotactic molecules would be released from the mucin solutions that were used in the in vitro assay in the current study, although B. pilosicoli is known to possess membrane-associated serine proteases and other proteases that could be involved in their degradation and release (Dassanayake et al., 2004).

The higher concentrations of mucin that were used here were highly viscous (Table 2), and these concentrations had 
similar viscosities to those that were found in the mucus layer overlying the epithelium in the porcine caecum and colon. Hence, the behaviour of the strains at the higher mucin concentrations is likely to reflect their activities in vivo, when they encounter the mucus barrier. This relationship led to an investigation of the potential involvement of the viscosity of the mucin solutions in their attractiveness to the spirochaetes. Previously, a strain of the spirochaete Leptospira interrogans (biflex) was shown to possess a 'viscotaxis' response, whereby it was attracted to a viscosity gradient made up of different concentrations of the viscous linear polymer PVP (Petrino \& Doetsch, 1978). When B. pilosicoli 95/1000 was tested against dilutions of PVP with different viscosities, it showed a response that resembled its response to mucin, although the $R_{\text {att }}$ values were numerically considerably less than those achieved with mucin at a given viscosity. Hence, it appears that at least part of the response of B. pilosicoli to mucin is likely to be a form of viscotaxis. In future work it would be interesting to add chemotactic materials from mucin to the PVP solutions, to see whether the combinations would increase the overall $R_{\text {att }}$ to levels similar to those achieved with mucin. It is not clear how a viscotaxis response could function, but $B$. pilosicoli strain NK1f has been shown to develop improved motion efficiency with increasing viscosity of a motility medium (Nakamura et al., 2006). Hence it is possible that the spirochaete cells that entered the viscous solutions through normal motility developed improved motion efficiency in the solutions, and then travelled up the tubes. In this way, at least part of the observed 'viscotaxis' may be simply due to increases in motion efficiency in response to increasing viscosity of the medium.

It was interesting that B. hyodysenteriae strain B204 showed a somewhat different pattern of 'attraction' to mucin, with this phenomenon declining at higher mucin concentrations. In explanation, initially it was thought that $B$. pilosicoli and B. hyodysenteriae might differ in their changes in motion efficiency relative to the viscosity, perhaps related to the fact that $B$. pilosicoli strains have 8-12 periplasmic flagella per cell, whilst $B$. hyodysenteriae strains have 22-28 (Stanton, 2006). When B204 was tested with the PVP solutions, however, its $R_{\mathrm{att}}$ values continued to increase as the viscosity increased. It appears therefore that factors other than viscosity may affect the behaviour of $B$. hyodysenteriae in high concentrations of mucin. For example, at high mucin concentrations the response to the chemoattractant properties of mucin may be switched off, such that only the viscotaxis effects still operate. This would then explain why, at a viscosity of around $14 \mathrm{mPa} \mathrm{s}$, the $R_{\mathrm{att}}$ for $\mathrm{B} 204$ had declined in mucin to a value similar to that found in PVP (Fig. 3).

The apparent viscotaxis of the spirochaetes was of practical interest and application, as different dietary ingredients and/or the addition of carboxymethylcellulose to experimental pig diets have been shown to influence the viscosity of the contents in the large intestine, with higher viscosities being associated with greater colonization by $B$. pilosicoli
(Hampson et al., 2000; Hopwood et al., 2002; Lindecrona et al., 2004). Hence there may be a link between viscosity of the colonic contents and the propensity for colonization with B. pilosicoli and B. hyodysenteriae. Such a link would also be important in disease states, where the composition or viscosity of the mucus layer might change. For example, in swine dysentery there is a great outpouring of mucus from the colonic crypts in response to the presence of $B$. hyodysenteriae. Depending on the composition and viscosity of this material, it might either enhance or inhibit colonization by this spirochaete species. The differences in response to mucin by the two pathogenic species also may help to account for the different behaviours and colonization sites for the two species. B. hyodysenteriae is found in the lumen of the colon and deep in the colonic crypts, where it enters goblet cells. Although B. pilosicoli also can enter the crypts, unlike $B$. hyodysenteriae it specifically penetrates the dense mucus layer at the crypt shoulders, and attaches to the underlying colonic enterocytes (Jensen et al., 2000). Presumably, as the mucus concentration increases towards the enterocyte surface, the attraction to mucin and the motion efficiency of B. pilosicoli would continue to increase, and this may be the means by which the subsequent specific attachment to the cell surface is facilitated. On the other hand, the densest part of the mucus barrier at the cell surface might be less attractive to $B$. hyodysenteriae, such that it does not undergo polar attachment.

\section{ACKNOWLEDGEMENTS}

R.N. was in receipt of a postgraduate scholarship from Murdoch University. The authors gratefully acknowledge Drs Nyree Phillips, Tom La, Jae Cheol Kim, Jung Min Heo and Judy Robertson for their technical assistance and advice.

\section{REFERENCES}

Bellgard, M. I., Wanchanthuek, P., La, T., Ryan, K., Moolhuijzen, P., Albertyn, Z., Shaban, B., Motro, Y., Dunn, D. S. \& other authors (2009). Genome sequence of the pathogenic intestinal spirochete Brachyspira hyodysenteriae reveals adaptations to its lifestyle in the porcine large intestine. PLoS One 4, e4641.

Dassanayake, R. P., Caceres, N. E., Sarath, G. \& Duhamel, G. E. (2004). Biochemical properties of membrane-associated proteases of Brachyspira pilosicoli isolated from humans with intestinal disorders. J Med Microbiol 53, 319-323.

Greenberg, E. P. \& Canale-Parola, E. (1977). Chemotaxis in Spirochaeta aurantia. J Bacteriol 130, 485-494.

Hampson, D. J. \& Duhamel, G. E. (2006). Porcine colonic spirochetosis/intestinal spirochetosis. In Diseases of Swine, 9th edn, pp. 755-767. Edited by B. E. Straw, J. J. Zimmerman, S. D’Allaire, \& D. J. Taylor. Oxford: Blackwell Publishing.

Hampson, D. J., Robertson, I. D., La, T., Oxberry, S. L. \& Pethick, D. W. (2000). Influences of diet and vaccination on colonisation of pigs with the intestinal spirochaete Brachyspira (Serpulina) pilosicoli. Vet Microbiol 73, 75-84.

Hampson, D. J., Oxberry, S. L. \& La, T. (2006). Potential for zoonotic transmission of Brachyspira pilosicoli. Emerg Infect Dis 12, 869-870. 
Hopwood, D. E., Pethick, D. W. \& Hampson, D. J. (2002). Increasing the viscosity of the intestinal contents stimulates proliferation of enterotoxigenic Escherichia coli and Brachyspira pilosicoli in weaner pigs. Br J Nutr 88, 523-532.

Jensen, T. K., Møller, K., Boye, M., Leser, T. D. \& Jorsal, S. E. (2000). Scanning electron microscopy and fluorescent in situ hybridization of experimental Brachyspira (Serpulina) pilosicoli infection in growing pigs. Vet Pathol 37, 22-32.

Johansson, M. E., Phillipson, M., Petersson, J., Velcich, A., Holm, L. \& Hansson, G. C. (2008). The inner of the two Muc2 mucin-dependent mucus layers in the colon is devoid of bacteria. Proc Natl Acad Sci U S A 105, 15064-15069.

Kennedy, M. J. \& Yancey, R. J. (1996). Motility and chemotaxis in Serpulina hyodysenteriae. Vet Microbiol 49, 21-30.

Kunkle, R. A., Harris, D. L. \& Kinyon, J. M. (1986). Autoclaved liquid medium for propagation of Treponema hyodysenteriae. J Clin Microbiol 24, 669-671.

Law, C. L. H., Grierson, J. M. \& Stevens, S. M. B. (1994). Rectal spirochaetosis in homosexual men: the association with sexual practices, HIV infection and enteric flora. Genitourin Med 70, 26-29.

Li, C., Motaleb, A., Sal, M., Goldstein, S. F. \& Charon, N. W. (2000). Spirochete periplasmic flagella and motility. $J$ Mol Microbiol Biotechnol 2, 345-355.

Lidell, M. E., Moncada, D. M., Chadee, K. \& Hansson, G. C. (2006). Entamoeba histolytica cysteine proteases cleave the MUC2 mucin in its C-terminal domain and dissolve the protective colonic mucus gel. Proc Natl Acad Sci U S A 103, 9298-9303.

Lindecrona, R. H., Jensen, T. K. \& Møller, K. (2004). Influence of diet on the experimental infection of pigs with Brachyspira pilosicoli. Vet Rec 154, 264-267.

Lux, R., Moter, A. \& Shi, W. (2000). Chemotaxis in pathogenic spirochetes: directed movement towards targeting tissues? J Mol Microbiol Biotechnol 2, 355-364.

Margawani, K. R., Robertson, I. D., Brooke, C. J. \& Hampson, D. J. (2004). Prevalence, risk factors and molecular epidemiology of Brachyspira pilosicoli in humans on the island of Bali, Indonesia. $J$ Med Microbiol 53, 325-332.

Milner, J. A. \& Sellwood, R. (1994). Chemotactic response to mucin by Serpulina hyodysenteriae and other porcine spirochetes: potential role in intestinal colonization. Infect Immun 62, 4095-4099.

Moulton, R. C. \& Montie, T. C. (1979). Chemotaxis by Pseudomonas aeroginosa. J Bacteriol 137, 274-280.

Munshi, M. A., Traub, R. J., Robertson, I. D., Mikosza, A. S. \& Hampson, D. J. (2004). Colonization and risk factors for Brachyspira aalborgi and Brachyspira pilosicoli in humans and dogs on tea estates in Assam, India. Epidemiol Infect 132, 137-144.

Nakamura, S., Adachi, Y., Goto, T. \& Magariyama, Y. (2006). Improvement in motion efficiency of the spirochete Brachyspira pilosicoli in viscous environments. Biophys J 90, 3019-3026.
Petrino, M. G. \& Doetsch, R. N. (1978). 'Viscotaxis', a new behavioural response of Leptospira interrogans (biflexa) strain B16. J Gen Microbiol 109, 113-117.

Stanton, T. B. (2006). The genus Brachyspira. In The Prokaryotes, vol. 7, pp. 330-356. Edited by S. Falkow, E. Rosenberg, K. H. Schleifer \& E. Stackebrandt. New York: Springer.

Stephens, C. P. \& Hampson, D. J. (2001). Intestinal spirochaetes infection of chickens: a review of disease associations, epidemiology and control. Anim Health Res Rev 2, 83-91.

Stephens, C. P. \& Hampson, D. J. (2002). Experimental infection of broiler breeder hens with the intestinal spirochaete Brachyspira (Serpulina) pilosicoli causes reduced egg production. Avian Pathol 31, 169-175.

Taylor, D. J., Simmons, J. R. \& Laird, H. M. (1980). Production of diarrhoea and dysentery in pigs by feeding pure cultures of a spirochaete differing from Treponema hyodysenteriae. Vet Rec 106, 326-332.

Trivett-Moore, N. L., Gilbert, G. L., Law, C. L. H., Trott, D. J. \& Hampson, D. J. (1998). Isolation of Serpulina pilosicoli from rectal biopsy specimens showing evidence of intestinal spirochetosis. J Clin Microbiol 36, 261-265.

Trott, D. J., McLaren, A. J. \& Hampson, D. J. (1995). Pathogenicity of human and porcine intestinal spirochetes in day-old specificpathogen-free chicks: an animal model of intestinal spirochetosis. Infect Immun 63, 3705-3710.

Trott, D. J., Huxtable, C. R. \& Hampson, D. J. (1996). Experimental infection of newly weaned pigs with human and porcine strains of Serpulina pilosicoli. Infect Immun 64, 4648-4654.

Trott, D. J., Combs, B. G., Oxberry, S. L., Mikosza, A. S. J., Robertson, I. D., Passey, M., Taime, J., Sehuko, R. \& Hampson, D. J. (1997a). The prevalence of Serpulina pilosicoli in humans and domestic animals in the Eastern Highlands of Papua New Guinea. Epidemiol Infect 119, 369-379.

Trott, D. J., Jensen, N. S., Saint Girons, I., Oxberry, S. L., Stanton, T. B., Lindquist, D. \& Hampson, D. J. (1997b). Identification and characterisation of Serpulina pilosicoli isolates from the blood of critically-ill patients. J Clin Microbiol 35, 482-485.

Trott, D. J., Mikosza, A. S. J., Combs, B. G., Oxberry, S. L. \& Hampson, D. J. (1998). Population genetic analysis of Serpulina pilosicoli and its molecular epidemiology in villages in the Eastern Highlands of Papua New Guinea. Int J Syst Bacteriol 48, 659-668.

Witters, N. A. \& Duhamel, G. E. (1999). Motility-regulated mucin association of Serpulina pilosicoli, the agent of colonic spirochetosis of humans and animals. Adv Exp Med Biol 473, 199-205.

Zhang, P., Cheng, X. \& Duhamel, G. E. (2000). Cloning and DNA sequence analysis of an immunogenic glucose-galactose MglB lipoprotein homologue from Brachyspira pilosicoli, the agent of colonic spirochetosis. Infect Immun 68, 4559-4565.

Edited by: P. W. O'Toole 